\title{
Erratum to: Quiver varieties and Demazure modules
}

\section{Alistair Savage}

Published online: 14 March 2010

(C) Springer-Verlag 2010

Erratum to: Math. Ann. (2006) 335:31-46 DOI 10.1007/s00208-005-0694-7

Part of the proofs of Proposition 6.1 and Theorem 8.1 relied on the statement that if $w=r_{i_{l}} \cdots r_{i_{1}}$ is a reduced expression of an element of the Weyl group and $X \in B_{w}(\mathbf{d})$, then $X=\tilde{e}_{i_{1}}^{n_{1}} \ldots \tilde{e}_{i_{l}}^{n_{l}} X_{w}$ for some $n_{1}, \ldots, n_{l} \in \mathbb{Z}_{\geq 0}$. In fact, this is not true. A counterexample can be found in [1, Example 4.6.3]. However, Propositions 6.1 and 8.1 still hold. Arguments avoiding the above claim are presented below. We thank H. Nakajima and S. Naito for bringing the error to our attention.

\section{Corrected proof of Proposition 6.1}

We first prove that for all $X \in B_{w}(\mathbf{d}), X$ consists of (orbits of) subrepresentations (up to isomorphism) of $\left(x_{w}, t_{w}\right)$. Our proof is by induction on the length of $w$. If this length is zero, the statement is trivial. Let $w=r_{i_{l}} \cdots r_{i_{1}}$ be a reduced expression and $X \in B_{w}(\mathbf{d})$. If $X_{\lambda_{\mathbf{d}}}=\mathfrak{L}(0, \mathbf{d})$ then, by (1.2), we have

$$
X=\tilde{f}_{i_{l}}^{n_{l}} \cdots \tilde{f}_{i_{1}}^{n_{1}} X_{\lambda_{\mathbf{d}}}
$$

The online version of the original article can be found under doi:10.1007/s00208-005-0694-7.

This research was supported in part by the Natural Sciences and Engineering Research Council (NSERC) of Canada.

A. Savage $(\varangle)$

Department of Mathematics and Statistics, University of Ottawa, Ottawa, ON, Canada

e-mail: alistair.savage@uottawa.ca 
for some $n_{1}, \ldots, n_{1} \in \mathbb{Z}_{\geq 0}$. Let

$$
X^{\prime}=\tilde{e}_{i_{l}}^{n_{l}} X=\tilde{f}_{i_{l-1}}^{n_{l-1}} \cdots \tilde{f}_{i_{1}}^{n_{1}} X_{\lambda_{\mathbf{d}}} .
$$

Since $r_{i_{l}} w=r_{i_{l-1}} \cdots r_{i_{1}}$ is a reduced expression, we see that $X^{\prime} \in B_{r_{i_{l}} w}(\mathbf{d})$ by (1.2). It follows from the inductive hypothesis that $X^{\prime}$ consists of (orbits of) subrepresentations (up to isomorphism) of $\left(x_{r_{i_{l}} w}, t_{r_{i_{l}} w}\right)$. Let

$$
X_{w}=\left\{\left[x_{w}, t_{w}\right]\right\} \in B_{w}(\mathbf{d}), \quad X_{r_{i_{l}} w}=\left\{\left[x_{r_{i_{l}} w}, t_{r_{i_{l}} w}\right]\right\} \in B_{r_{i_{l}} w}(\mathbf{d}) .
$$

Let $\mathbf{V}, \mathbf{V}^{\prime}, \mathbf{V}^{w}$ and $\mathbf{V}^{r_{l}}{ }^{w}$ be the spaces corresponding to the representations whose orbits are points of $X, X^{\prime}, X_{w}$ and $X_{r_{i}{ }}$ respectively. By the definition of the crystal operators in [2], we have $\mathbf{V}_{i}^{w}=\mathbf{V}_{i}^{r_{i}{ }^{w}}$ for $i \neq i_{l}$ and

$$
\mathbf{V}_{i_{l}}^{w} \stackrel{\left(\left(x_{w}\right)_{h}, t_{w}\right)}{\cong} \operatorname{ker}\left(\bigoplus_{h: \operatorname{in}(h)=i_{l}} \mathbf{V}_{\text {out }(h)}^{r_{i_{l}} w} \oplus \mathbf{W}_{i_{l}} \stackrel{\left(\varepsilon(h)\left(x_{r_{l}} w\right)_{h}, 0\right)}{\longrightarrow} \mathbf{V}_{i_{l}}^{r_{i_{l}} w}\right) \stackrel{\text { def }}{=} \tilde{K}
$$

Since $X=\tilde{f}_{i_{l}}^{n_{l}} X^{\prime}$, by the definition of the crystal operators given in [2] we have

$$
\mathbf{V}_{i}=\mathbf{V}_{i}^{\prime}, i \neq i_{l}, \quad \text { and } \quad \mathbf{V}_{i_{l}} \cong \mathbf{V}_{i_{l}}^{\prime} \oplus \mathbb{C}^{n_{l}}
$$

and an open dense subset of $X$ consists of orbits of representations $(x, t)$ such that $\mathbf{V}^{\prime}$ is $x$-stable and $\left[x^{\prime}, t^{\prime}\right] \in X^{\prime}$ where $x^{\prime}=\left.x\right|_{\mathbf{V}^{\prime}}, t^{\prime}=\left.t\right|_{\mathbf{V}^{\prime}}$. Now, by the stability and moment map conditions, we have that the map

$$
\mathbf{V}_{i_{l}} \stackrel{\left(x_{h}, t\right)}{\hookrightarrow} \operatorname{ker}\left(\bigoplus_{h: \text { in }(h)=i_{l}} \mathbf{V}_{\text {out }(h)}^{\prime} \oplus \mathbf{W}_{i_{l}} \stackrel{\left(\varepsilon(h) x_{h}^{\prime}, 0\right)}{\longrightarrow} \mathbf{V}_{i_{l}}^{\prime}\right) \stackrel{\text { def }}{=} K
$$

is injective. Since $\left(x^{\prime}, t^{\prime}\right)$ is a subrepresentation (up to isomorphism) of $\left(x_{r_{i_{l}} w}, t_{r_{i_{l}} w}\right)$, we have (after replacing representations by different orbit representatives if necessary) $K \subseteq \tilde{K}$. Thus, for $(x, t)$ in an open dense subset of $X,(x, t)$ is isomorphic to a subrepresentation of $\left(x_{w}, t_{w}\right)$ by (1.1), (1.2) and the inductive hypothesis. As in the original proof, we see that the set of orbits of subrepresentations of $\left(x_{w}, t_{w}\right)$ is closed and thus $X$ consists entirely of such representations.

The proof of the converse statement, that if every point of $X$ is an orbit of a subrepresentation of $\left(x_{w}, t_{w}\right)$ then $X \in B_{w}(\mathbf{d})$, is as in the original.

\section{Corrected proof of Theorem 8.1}

We prove the case of $w_{n}^{-}$. The case of $w_{n}^{+}$is analogous. By (1.2),

$$
P_{w_{n}^{-}}(\lambda)=\left\{\tilde{f}_{i_{n}}^{k_{n}} \cdots \tilde{f}_{i_{1}}^{k_{1}} P_{\lambda} \mid k_{1}, \ldots, k_{n} \in \mathbb{Z}_{\geq 0}, i_{j} \equiv j \quad \bmod 2\right\} .
$$


Suppose $P \in P_{w_{n}^{-}}(\lambda)$. Since the blocks of any stack in a Young pyramid must alternate color, we see that the maximum height of any stack in $P$ is $n$ and the maximum height of any stack with bottom block of color 0 (e.g. the stacks in the column one) is $n-1$. Since $P$ is 1-reduced, the heights of stacks must strictly decrease as we move east. Therefore, the height of any stack in the $i$ th column is less than or equal to $n-i$. Therefore $P$ is a subpyramid of $P_{\lambda}^{w_{n}^{-}}$.

The proof of the converse statement, that any subpyramid of $P_{\lambda}^{w_{n}^{-}}$is contained in $P_{w_{n}^{-}}(\lambda)$, is as in the original.

\section{References}

1. Naito, S., Sagaki, D.: Mirković-Vilonen polytopes lying in a Demazure crystal and an opposite Demazure crystal (2008, Preprint). arXiv:0806.3112v2 [math.QA]

2. Saito, Y.: Crystal bases and quiver varieties. Math. Ann. 324(4), 675-688 (2002) 